\title{
Durability of the partial reinforcement and partial delay of reinforcement extinction effects after minimal acquisition training
}

\author{
MITRI E. SHANAB* and DANA W. BIRNBAUM \\ California State University, Fresno, California 93740
}

\begin{abstract}
Four groups of 10 rats each were given six acquisition trials (Phase 1) under continuous reinforcement (CR), partial reinforcement (PR), constant delay (CD), or partial delay of reinforcement (PD) conditions. In Phase 2, all Ss were given 18 nonreinforced trials, followed by 12 continuously reinforced trials in Phase 3. In Phase 4, all Ss were given 12 more extinction trials. A constant 24-h ITI was observed throughout the experiment. A strong partial reinforcement extinction effect (PREE) was obtained in both Phases 2 and 4 . Only a temporary partial delay of reinforcement effect (PDRE) was observed, which was restricted to the first nine trials of the first extinction phase. No constant delay of reinforcement effect (CDRE) was observed in either extinction phase. The results were discussed in terms of both frustration and sequential theories.
\end{abstract}

The partial reinforcement extinction effect (PREE) following minimal acquisition is quite a reliable phenomenon, in that several studies have shown that Ss receiving limited acquisition training (two to six trials) under a partial reinforcement (PR) schedule are more resistant to extinction than comparable Ss trained on a continuous reinforcement (CR) schedule (Robbins, 1971). There are two studies in the literature that attempted to extend this finding to extinction following minimal acquisition training under either partial delay (PD) or constant delay of reinforcement (CD). After five acquisition trials, McCain and Bowen (1967) found that Ss delayed on each acquisition trial were more resistant to extinction than Ss immediately reinforced on each trial. Howlett and Sheldon (1968) compared partial delay of reinforcement with continuous reinforcement conditions after only three acquisition trials. They reported that the PD Ss were more resistant to extinction than the CR Ss. In both studies, extinction trials were given in one session. In the Howlett and Sheldon study, which used sucrose reinforcement (of unspecified concentration), both acquisition and extinction trials were massed and given on the same day. Moreover, the delay was shifted from a $60-\mathrm{sec}$ interval in acquisition to a $5-\mathrm{sec}$ interval in extinction. The latter procedure raises a question as to whether the shift in delay alone rather than the differential reinforcement treatment given in acquisition was responsible for the obtained differences in extinction. In neither study was a partial reinforcement group included, despite the obvious theoretical and empirical relevance of such a group. At any rate, the evidence for a partial delay of reinforcement effect (PDRE) or a constant delay of reinforcement effect (CDRE) following a small number

* Reprint requests should be addressed to Mitri E. Shanab, Department of Psychology, California State University, Fresno, California 93740 . of acquisition trials is at best minimal. Therefore, one purpose of the study was to run a comprehensive design that included both partial delay and constant delay of reinforcement as well as partial and continuous reinforcement conditions, in the hope of replicating and possibly extending the results of the above two studies.

The second purpose of the study was to test certain implications of both Amsel's frustration theory (1967) and Capaldi's sequential theory (1967). The former theory leads to the prediction that, as long as the anticipatory frustration, or persistence, mechanism $\left(\mathrm{r}_{\mathrm{F}}-\mathrm{S}_{\mathrm{F}}\right)$ remains intact in the sense that it does not undergo extinction, a sustained partial reinforcement or partial delay of reinforcement extinction effect will be obtained following interpolated continuous reinforcement training. This prediction has received support from several studies in which extended acquisition training was given (Surridge, Mock, \& Amsel, 1968; Traupmann, Wong, \& Amsel, 1971; Amsel, Wong, \& Traupmann, 1971). Following minimal acquisition, frustration theory also predicts a sustained PREE as long as the reinforcer given in acquisition is in the form of multiple pellets (cf. Amsel, Hug, \& Surridge, 1968). According to this view, a trial with multiple pellets is functionally equivalent to many trials with a single pellet. Traupmann and Wong (1971), using multiple pellets, gave rats only four acquisition trials under either a partial (NRNR sequence) or a continuous reinforcement schedule and tested them in extinction after they had received 16 continuously reinforced trials interpolated between acquisition and extinction. The PR Ss were more resistant to extinction than the CR Ss.

Capaldi's sequential theory (1967) usually leads to similar predictions as the frustration theory. The sequential theory assumes that the stimulus aftereffects of nonreinforced $\left(\mathrm{S}^{\mathrm{N}}\right)$ as well as delayed $\left(\mathrm{S}^{\mathrm{D}}\right)$ trials become conditioned to the instrumental response on 
reinforced trials. Since CR Ss never experience either nonreinforcement or delay of reinforcement during acquisition, they would show less resistance to extinction than Ss receiving either condition because they experience more generalization decrement in extinction. By the same reasoning, if the connection between $S^{N}$ or $S^{D}$ and the instrumental response remains somewhat intact in a first extinction phase, then the PREE or PDRE would be expected to occur in a second extinction phase following an interpolated block of continuously reinforced trials. Thus, both the frustration and the sequential theories lead to the same predictions concerning the PREE and PDRE. However, with constant delay, the frustration theory predicts no extinction differences between a constant delay of reinforcement (CD) and a continuous reinforcement (CR) group, on the assumption that no frustration would be experienced by CD Ss during acquisition, especially with few acquisition trials; therefore, no CDRE would be expected (cf. Surridge et al, 1968). On the other hand, the sequential theory would predict that CD Ss would persist longer in extinction than the CR Ss, since the former Ss would suffer less of a generalization decrement than the latter Ss.

\section{Design \\ METHOD \\ The study consisted of four phases. In Phase 1 a 2 by 2 factorial design was used in which different $S$ s received continuous reinforcement (CR), constant delay of reinforcement (CD), partial reinforcement (PR), or partial delay of reinforcement (PD) conditions for six trials. In Phase 2 all Ss were given 18 extinction trjals. In Phase 3 all Ss were given 12 trials of continuous reinforcement, and in Phase 4 all Ss were given 12 more extinction trials. All training was given at one trial per day.}

\section{Subjects}

The Ss were 40 male albino rats of the Sprague-Dawley strain, approximately 90 days old at the beginning of the experiment.

\begin{abstract}
Apparatus
A $1.9-\mathrm{m}$ L-shaped wooden runway was used. The entire runway was covered with Plexiglas. The width and height of all sections of the runway were 9.2 and $12.1 \mathrm{~cm}$, respectively. The goalbox, which was at right angles to the runway, was $15.2 \mathrm{~cm}$ long. Except for the final $27.9 \mathrm{~cm}$ that served as the delay chamber, the entire runway floor had stainless steel grids placed $1.3 \mathrm{~cm}$ apart. The startbox was $28.9 \mathrm{~cm}$ long. The goalbox was painted white, while the rest of the runway was painted black. Three guillotine doors were used: The first separated the startbox from the alley, the second separated the alley from the delay chamber, and the third separated the delay chamber from the goalbox. Two sets of photocells were installed in the runway; the first was located $2.54 \mathrm{~cm}$ outside the startbox door, while the second was located $12.7 \mathrm{~cm}$ inside the delay chamber. Interruption of the two photobeams started and stopped a Standard Electric timer that measured total running time over a distance of $1.4 \mathrm{~m}$.
\end{abstract}

\section{Procedure}

Uppon arrival from the supplier, Ss were placed on free feeding for 10 days. They were then maintained on a daily ration of $10 \mathrm{~g}$ of Purina Lab Chow and had free access to water throughout the experiment. During the first week of deprivation, each $S$ was handled and allowed to eat $45-\mathrm{mg}$ Noyes food pellets from the porcelain foodcup later used in the goalbox. Following this handling period, each $S$ was allowed to explore the unbaited runway for $1 \mathrm{~min} /$ day for 10 days. During this period, all doors were raised and all circuitry was turned on to adapt the $S$ to the various noises of the equipment.

Following exploration, all Ss were given acquisition training at one trial per day for 6 days. The sequence of reward and nonreward or delayed reward was the same for both PR and PD Ss. Specifically, Ss in both groups first received two reinforced (R) trials, followed by three nonreinforced (N) or delayed (D) trials, while the last trial was an immediately reinforced trial. The reinforcer consisted of 2245 -mg pellets. Where applicable, proper adjustments were made for food eaten in the apparatus to equate drive level. The $\mathrm{S}$ was confined in the delay chamber on either $\mathrm{N}$ or $\mathrm{D}$ trials for $20 \mathrm{sec}$. On all trials, as soon as the $\mathrm{S}$ broke the last photobeam, the guillotine door was lowered to prevent retracing. On $\mathrm{N}$ trials an empty foodcup was placed in the goalbox, while on D trials the baited foodcup was placed in the box after the 20-sec confinement period.

In Phase 2 all Ss received one nonreinforced trial a day for 18 days. In Phase 3 all Ss received one immediately reinforced trial a day for 12 days. In Phase 4 all Ss received one nonreinforced trial a day for 12 days. In both extinction phases the $S$ was confined in the goalbox for $20 \mathrm{sec}$ and in neither phase was the foodcup present in the goalbox.

\section{RESULTS}

All analyses are based on total speed, which refers here to the reciprocal of total time.

\section{Phase 1}

As can be seen in Fig. 1, CR Ss were running faster than either the PD, PR, or CR Ss by the end of Phase 1. A two-way analysis of variance test on the mean total speed over the entire phase yielded nonsignificant main effects (both $F s<1$ ) and a nonsignificant interaction effect $[F(1,36)=3.40, p>.05]$. However, an analysis of variance performed on the last trial in acquisition yielded a significant Schedule by Delay interaction $[F(1,36)=6.31, p<.05]$. Neither the delay nor the schedule effect was significant $[F(1,36)=1.25, p>.05$ for schedule and $F(1,36)=1.20, p>.05$ for delay] Individual comparisons using Duncan's multiple range test showed that CR Ss ran significantly faster than either the PR or CD Ss $(p<.05)$. The CR-PD comparison was not significant $(\mathrm{p}>.05)$.

\section{Phase 2}

As Fig. 1 shows, the PR Ss ran faster than the CR Ss during extinction, in spite of the fact that the latter were running significantly faster at the end of acquisition. Moreover, it seems clear that the PR Ss were faster than the other two groups throughout this phase. It is interesting to note that the CD group was the fastest to extinguish.

An analysis of variance test using trials as the within-Ss variable was performed on the mean speeds over Trials 1-9, yielding significant schedule and delay effects $[F(1,36)=31.96, p<.001$ and $F(1,36)=13.64$, $\mathrm{p}<.005$, respectively]. The interaction of the two 


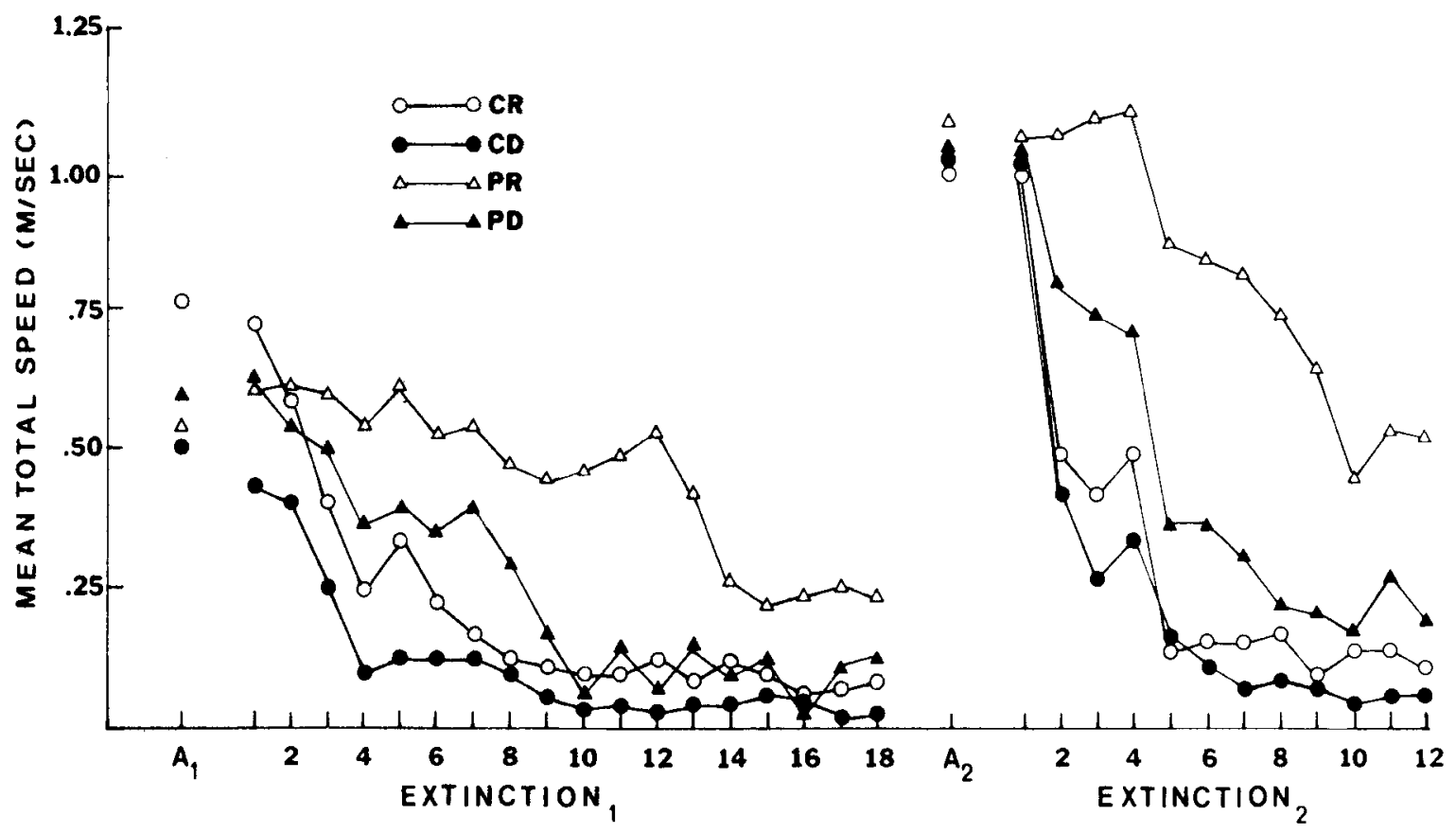

TR IALS

Fig. 1. Mean total speed for all four experimental groups over the first acquisition and extinction phases as well as the second acquisition and extinction phases.

variables was not significant $(\mathrm{F}<1)$. The trials effect was highly significant $[F(8,288)=32.6, p<.001]$. Both the Schedule by Trials and the Schedule by Delay by Trials interaction effects were significant $[\mathrm{Fs}(8,288)=$ 4.00 and 3.01 , both ps $<.01$ \}. However, the Delay by Trials interaction was not significant. Individual comparisons using Duncan's multiple range test revealed that Group PR ran significantly faster than both Groups $C R$ and CD $(p<.01)$, as well as Group PD $(p<.05)$. The PD Ss ran significantly faster than the CD Ss $(p<.01)$. Similarly, the CR Ss ran faster than the CD Ss $(\mathrm{p}<.05)$.

Because of the triple interaction and the interaction of trials with schedule, $F$ tests of simple effects were performed to evaluate the rate of performance decrement during this part of the phase. It was found that Group CR showed a greater performance decrement than Group PR $[\mathrm{F}(8,288)=4.21, \mathrm{p}<.01]$, reflecting a significant PREE. Similarly, a significant PDRE was revealed by the PD-CR comparison $[F(8,288)=2.15$, $\mathrm{p}<.05]$. No CDRE was obtained, in that both the CR and $\mathrm{CD}$ groups seemed to have extinguished at the same rate during the first nine trials of Phase $2[F(8,288)=$ $1.80, \mathrm{p}>.05]$. The $\mathrm{PR}$ extinguished at a slower rate than either the PD group $[F(8,288)=2.02, p<.05]$ or the $\mathrm{CD}$ group $[\mathrm{F}(8,288)=2.54, \mathrm{p}<.025]$. No difference in performance decrement was obtained between the $\mathrm{CD}$ and PD groups $(\mathrm{F}<1)$.

A similar analysis of variance with repeated measures was performed on the mean speeds over Trials 10-18, revealing significant main effects $[\mathrm{Fs}(1,36)=61.43$ and 50.84 , both ps $<.001$ for schedule and delay, respectively]. The Schedule by Delay interaction was also significant $[F(1,36)=19.81, p<.001]$. The trials effect as well as the Schedule by Trials and the Delay by Trials interaction effects were significant $[F s(8,288)=$ $4.66,3.19$, and 3.82 , all $\mathrm{ps}<.01$, respectively]. The triple interaction was also significant $[F(8,288)=2.56$, $\mathrm{p}<.05]$. The Duncan's test showed that the PR Ss were still running faster than the other three groups $(\mathrm{p}<.01)$. The PD Ss, however, did not run significantly faster than the CR Ss, nor did the latter run faster than the CD Ss. The results of the analysis of the groups' performance decrement over Trials 10-18 revealed no difference between either the $C R$ and $C D$ or the PD and $C D$ groups (both $F_{S}<1$ ), indicating the absence of either a CDRE or a PDRE. However, a significant PREE was obtained in that the CR Ss showed a significantly greater rate of performance decrement than the PR Ss $[F(8,288)=$ $5.34, p<.001]$. Not only did the PR group show less performance decrement relative to the CR group, but also to both the CD and PD groups $[F s(2,288)=6.37$ and 6.18 , both ps $<.001$, respectively].

Finally, an analysis of variance test was performed on the mean speeds over the last trial of Phase 2, yielding significant schedule and delay effects $[F(1,36)=11.97$, $p<.01$ and $F(1,36)=6.71, p<.05$, respectively]. The Schedule by Delay interaction was not significant $[F(1,36)=1.48, p>.05]$. Individual comparisons using Duncan's test showed that Group PR ran faster than Groups CR and CD $(\mathrm{p}<.01)$, as well as Group PD $(p<.05)$. All other comparisons were not significant.

\section{Phase 3}

As Fig. 1 shows, all groups increased their running speed and reached comparable asymptotes by the end of 
the phase. An analysis of variance performed on the last three trials revealed no significant schedule, delay, or Schedule by Delay interaction effects (all Fs $<1$ ). Moreover, no significant trials, Delay by Trials, or Schedule by Delay by Trials interaction effects were found (all Fs $<1$ ). The Schedule by Trials interaction was also not significant $[F(2,72)=2.27, p>.05]$.

\section{Phase 4}

As can be seen in Fig. 1, the performance of the groups stabilized at different levels. An analysis of variance test, in which trials were included as the within-Ss variable, revealed significant schedule and delay effects $[F(1,36)=49.60, p<.001$ and $F(1,36)=$ $16.47, \mathrm{p}<.001$, respectively]. The Schedule by Delay interaction was also significant $[F(1,36)=7.85$, $\mathrm{p}<.01]$, as was the trials effect $[\mathrm{F}(11,396)=74.22$, $\mathrm{p}<.001]$. The Schedule by Trials, Delay by Trials, and Schedule by Delay by Trials interactions were all significant $[F(11,396)=7.42, p<.001, F(11,396)=$ $2.14, \mathrm{p}<.05$, and $\mathrm{F}(11,396)=2.56, \mathrm{p}<.01$, respectively].

Because of the multiple interactions obtained, separate trial by trial analysis of variance tests were performed over the first part of Phase 4. Trial 1 was excluded on the grounds that it could be considered an additional trial in Phase 3 , a fact supported by the obvious lack of difference between all four groups on this trial as well as on the last trial of Phase 3 . The results of the separate analysis of variance tests for Trials 2,3 , and 4 showed significant schedule effects $[\operatorname{Fs}(1,36)=30.30,32.83$, and 27.32 , all ps $<.001$. The delay effects were not significant for Trial 2, $F(1,36)=3.49, p>.05$, but were significant for Trials 3 and $4, F s(1,36)=5.52,7.54, p<.05$ and $p<.01$, respectively. No significant Schedule by Delay interaction was obtained for Trials 2,3 , or $4, \mathrm{Fs}(1,36)$ $=1.14,1.03$, and 1.31 , all ps $>.05$, respectively] . Individual comparisons using the Duncan test showed that the PR Ss ran significantly faster than the CR and CD Ss on Trials 2,3 , and $4(p<.01)$. The PR Ss also ran significantly faster than the PD Ss on Trials 2 and 3 $(\mathrm{p}<.05)$ and on Trial $4(\mathrm{p}<.01)$. The PD group ran significantly faster than the CR group on Trials 2 and 3 only $(p<.05)$. The PD Ss, on the other hand, ran significantly faster than the CD Ss on Trials 2, 3, and 4 $(\mathrm{p}<.01)$. Additionally, an analysis of variance performed on Trial 5' yielded significant main effects $[F s(1,36)=36.71$ and 11.22 , both ps $<.001$ for schedule and delay, respectively]. The Schedule by Delay interaction was also significant $[F(1,36)=12.49$, $\mathrm{p}<.001]$. Individual comparisons using Duncan's test showed that the PR group was still running significantly faster than either one of the other three groups $(p<.01)$. No other comparison was significant. This is further supported by an analysis of variance test with repeated measures over Trials 5-12. Both main effects and their interaction were significant $[F s(1,36)=47.22$, 20.12 , and 12.11 , all ps $<.001$ for schedule, delay, and
Schedule by Delay interaction, respectively]. The trials as well as the Schedule by Trials interaction effects were significant $[\mathrm{Fs}(7,252)=9.53$ and 5.11 , both ps $<.001]$. The Delay by Trials interaction was not significant $[F(7,252)=1.64, p>.05]$. However, the Schedule by Delay by Trials interaction was significant $[F(7,252)=$ $3.04, \mathrm{p}<.005]$. The results of the Duncan test again showed that the PR Ss ran significantly faster than all other Ss $(\mathrm{p}<.01)$. The PD Ss also ran significantly faster than the CD Ss $(p<.05)$. There was no significant difference between the PD and CR Ss, indicating the absence of a PDRE.

The results of the analysis of the rate of performance decrement over Trials 5-12 showed that the PR Ss decreased their speed at a significantly slower rate than either the CR, the CD, or the PD Ss $[F s(7,252)=7.67$, 5.71 , and 4.25 , all $\mathrm{ps}<.001$, respectively]. On the other hand, there was no significant performance decrement between the $P D$ and $C R$ groups $[F(7,252)=1.04$, $p>.05]$ or between the $C D$ and $C R$ groups $(F<1)$. The PD-CD difference was also not significant $(F<1)$.

As Fig. 1 indicates, all four groups reached stable speeds by the end of Phase 4 . This is supported by the results of an analysis of variance with repeated measures over Trials 10-12, which yielded a nonsignificant trials effect $[F(2,72)=1.24, p>.05]$. All interactions with trials were also not significant $(\mathrm{Fs}<1)$. However, the main effects of schedule and delay were significant $[F(1,36)=40.05, p<.001$ for schedule and $F(1,36)=$ $7.25, \mathrm{p}<.025$ for delay]. The Schedule by Delay interaction was not significant $[\mathrm{F}(1,36)=1.51$, $\mathrm{p}>$.05]

\section{DISCUSSION}

The present finding of a PREE which was sustained through an extinction phase that was followed by a block of continuously reinforced trials lends general support to the sustained PREE reported in the Traupmann and Wong study (1971), in which PR Ss given a block of continuously reinforced trials before extinction were more resistant to extinction than comparable CR Ss. More importantly, the present finding of an enduring PREE supports and extends the findings of both studies by Traupmann et al (1971) and Amsel et al (1971), in which identical results were obtained following extended acquisition training. These results are consistent with the multiple-pellet hypothesis (Amsel et al, 1968). According to this hypothesis, multiple pellets hasten the formation of the anticipatory reward mechanism $\left(r_{R}-S_{R}\right)$ so that $P R$ Ss receiving limited acquisition training also experience frustration and learn through the anticipatory frustration mechanism $\left(r_{F}-s_{F}\right)$ to make the instrumental response in the presence of frustration cues.

As noted earlier, the sequential hypothesis (1967) can also account for the enduring PREE following minimal acquisition training by making a similar assumption, namely, that the aftereffects of nonreinforcement (SN) 
are more strongly conditioned to the instrumental response in the PR than in the CR Ss. Thus, both hypotheses can account for the persistence of PR Ss in terms of the strong conditioning of the instrumental response to either frustration cues or cues occasioned by the aftereffects of nonreinforced trials.

The PDRE obtained in this study was weak and transient. In both this study and the Howlett and Sheldon study (1968), the PDRE lasted for nine extinction trials. Even after extended training, Surridge et al (1968) reported that the PDRE was present only during the initial 8 days of their first extinction phase. That the PDRE is relatively fragile is further demonstrated by the absence of a sustained PDRE in this as well as in the Surridge et al study (1968). In both studies the PDRE disappeared during the first extinction phase, which in frustration terms could be attributed to the extinction of the underlying $\mathrm{r}_{\mathrm{F}}-\mathrm{s}_{\mathrm{F}}$ mechanism, which in turn should lead to the abolition of the PDRE in a second extinction phase. The disappearance of the PDRE could similarly be attributed in sequential terms to the extinction of the already weak connection between the aftereffects of delay (SD) and the instrumental response.

The present results do not agree with those reported in McCain and Bowen's study (1967), in which a CDRE was obtained following a small number of acquisition trials. This discrepancy could be attributed to certain procedural differences between the two studies. For example, McCain and Bowen (1967) gave all acquisition or extinction trials in one session, while the present study gave one acquisition or extinction trial a day. Moreover, apparently in McCain and Bowen's study the Ss found an empty foodcup in the goalbox, whereas in this study no foodcup was present in the goalbox during extinction. However, the present finding of no CDRE is consistent with many studies that used extended acquisition training (cf. Campbell \& Knouse, 1972). Surridge et al (1968) report some unpublished findings which indicate that extinction following acquisition under a constant 30 -sec delay interval produces even less resistance to extinction than acquisition under continuous reinforcement conditions. Indirect support of the absence of a CDRE is to be found in several studies which showed that no evidence of learning is found under constant delay (Logan, 1960; Wike \& McWilliams, 1967). Moreover, Couch and Stanley (1967) found that Ss delayed $30 \mathrm{sec}$ on all trials performed at the same level as Ss receiving nonreinforcement all the time.

The absence of a CDRE in Phase 2 is consistent with the prediction made from Amsel's frustration theory (1967) that, since probably very little frustration is experienced by Ss receiving minimal acquisition under constant delay conditions, no extinction differences should be obtained between constant delay Ss and continuously reinforced Ss. On the other hand, these findings run counter to Capaldi's sequential theory (1967), which predicts a CDRE on the assumption that CD Ss experience less generalization decrement in extinction than the CR Ss. It could be argued, however, that because of the small number of acquisition trials and the fact that these trials were separated by long intervals $(I T I=24 \mathrm{~h})$, the aftereffects of delay $\left(\mathrm{S}^{\mathrm{D}}\right)$ in the constant delay group were only weakly conditioned to the instrumental response. Thus, in extinction the CD Ss would not experience significantly less generalization decrement than the CR Ss.

\section{REFERENCES}

Amsel, A. Partial reinforcement effects on vigor and persistence: Advances in frustration theory derived from a variety of within-subject experiments. In K. W. Spence and J. T. Spence (Eds.), The psychology of learning and motivation. Vol. 1. New York: Academic Press, 1967. Pp. 1-65.

Amsel, A., Hug, J. J., \& Surridge, C. T. Number of food pellets, goal approaches, and the partial reinforcement extinction effect after minimal acquisition. Journal of Experimental Psychology, 1968, 77, $530-534$.

Amsel, A., Wong, P. T. P., \& Traupmann, K. L. Short-term and long-term factors in extinction and durable persistence. Journal of Experimental Psychology, 1971, 90, 90-95.

Campbell, P. E., \& Knouse, S. B. Extinction following delayed reward: A review. Psychonomic Monograph Supplements, $1972,4(15$, Whole No. 63).

Capaldi, E. J. A sequential hypothesis of instrumental learning. In K. W. Spence and J. T. Spence (Eds.), The psychology of learning and motivation. Vol. 1. New York: Academic Press, 1967. Pp. 67-156

Couch, J. V., \& Stanley, L. R. Consistent delay of reward vs consistent nonreward in the alley. Psychonomic Science, $1967,9,497-498$.

Howlett, J. C., \& Sheldon, M. H. Effects of partial delay of reinforcement following a small number of acquisition trials. Psychonomic Science, 1968, 11, 259.

Logan, F. A. Incentive. New Haven: Yale University Press, 1960. McCain, G., \& Bowen, J. Pre- and post-reinforcement delay with a small number of acquisition trials. Psychonomic Science, 1967, 7, 121-122.

Robbins, D. Partial reinforcement: A selective review of the alleyway literature since 1960. Psychological Bulletin, 1971, $76,415-431$.

Surridge, C. T., Mock, K. R., \& Amsel, A. Effect of interpolated extinction after partial delay of reward training on subsequent reacquisition and extinction. Quarterly Joumal of Experimental Psychology, 1968, 20, 321-328.

Traupmann, K. L., \& Wong, P. T. P. The small-trial PREE and interpolated CRF training. Psychonomic Science, 1971, 23, 207-208.

Traupmann, K. L., Wong, P. T. P., \& Amsel, A. Durability of persistence as a function of number of partially reinforced trials. Journal of Experimental Psychology, 1971, 88, 372-375.

Wike, E. L., \& McWilliams, J. Duration of delay, delay-box confinement, and runway performance. Psychological Reports, $1967,21,865-870$.

(Received for publication September 24,1973 revision received November $8,1973$. 